\title{
A FORMULA FOR DEVIATION FROM COMMUTATIVITY: THE TRANSFER AND STEENROD SQUARES
}

\author{
RICHARD P. KUBELKA
}

\begin{abstract}
The ordinary cohomology transfer associated to the orbit space projection of a finite group action need not commute with stable cohomology operations. In particular, if an even group acts on a space, the resulting transfer $\tau$ will not generally commute with the Steenrod squares, $\mathrm{Sq}^{i}$. This paper contains a formula for the deviation from commutativity $\left(\mathrm{Sq}^{i} \tau-\tau \mathrm{Sq}^{i}\right) x$ in the case of an involution. The formula involves the restriction of $x$ to the cohomology of the fixed point set, as well as certain naturally occurring characteristic classes.
\end{abstract}

1. In 1968, Evens [4] gave a proof that the transfer map $\tau: H^{*}(H ; \mathbf{Z} / 2) \rightarrow$ $H^{*}(G ; \mathbf{Z} / 2)$ in group cohomology commutes with the Steenrod squaring operations $\mathrm{Sq}^{i}$, where $H$ is a subgroup of finite index in $G$. More recently, Becker, Gottlieb, Dold et al. have shown that transfers $\tau: h^{*}(E) \rightarrow h^{*}(B)$ exist for any cohomology theory $h^{*}(\quad)$ when, for example, $p: E \rightarrow B$ is a fibration with compact fibre. These transfers are induced by stable maps between the two spaces in the reverse direction and hence must commute with all stable cohomology operations, by naturality and stability. In particular, they commute with the $\mathrm{Sq}^{i}$.

When a finite group $G$ acts regularly on a finite simplicial complex $X$, we have a transfer $\tau: H^{*}(X, \mathbf{Z} / 2) \rightarrow H^{*}(X / G ; \mathbf{Z} / 2)$ in ordinary cohomology which comes from a chain-level map $\tau: C_{*}(X / G ; \mathbf{Z}) \rightarrow C_{*}(X ; \mathbf{Z})[3]$. If the action is free, then this $\tau$ is equal to the one that $\pi: X \rightarrow X / G$ possesses by virtue of its being a covering. It is a fact, however, $[7,6]$ that if $G$ does not act freely, then this transfer does not in general commute with Steenrod squares. In consequence, it cannot be induced by a stable map backwards as in the fibration case.

In this paper, we examine the case of a regular involution on a finite complex $X$ and derive a formula for the deviation from commutativity $\left(\tau \mathrm{Sq}^{i}-\mathrm{Sq}^{i} \tau\right)$. In particular, this applies to any smooth $\mathbf{Z} / 2$-action on a compact manifold $M$, by [5]. We also consider briefly the modification of this formula for regular actions of 2-groups on $X$. The results are taken from the author's dissertation [6], written under the direction of Professor Gregory Brumfiel at Stanford University. I would like to thank Professor Brumfiel for his insights and help. In addition, let me thank the referee for several useful suggestions.

Received by the editors March 9, 1981 and, in revised form, July 30, 1981. Presented at the annual meeting of the American Mathematical Society, January 9, 1981.

1980 Mathematics Subject Classification. Primary 55M35, 55S05, 55N 10.

Key words and phrases. Transfer, Steenrod squares, involution, fixed point set.

(C)1982 American Mathematical Society 0002-9939/82/0000-1077/\$02.50 
2. Suppose $\mathbf{Z} / 2$ acts regularly on the finite complex $K$. Then $k=K /(\mathbf{Z} / 2)$ is a complex and the orbit map $\pi: K \rightarrow k$ is simplicial [2]. Let $L$ be the fixed point set of the involution and $l=L /(\mathbf{Z} / 2) \approx L$. After suitable subdivision, we can find $n$, a regular neighborhood of $l$ in $k$ with deformation retraction $p^{\prime}: n \rightarrow l$. Then $N=\pi^{-1}(n)$ is an invariant regular neighborhood of $L$ in $K$. If we call $\dot{n}=n-l$ and $\dot{N}=N-L$, then $\pi \mid \dot{N}: \dot{N} \rightarrow \dot{n}$ is a double covering and it has a $\bmod 2$ characteristic class $\alpha \in H^{1}(\dot{n} ; \mathbf{Z} / 2)$. (Henceforth we assume that all coefficients are in $\mathbf{Z} / 2$.) Denote by $d^{*}$ the coboundary in the Mayer-Vietoris sequence of the triad $(k ; n, k-l)$ and let $\varphi: L \rightarrow K$ be the inclusion. Finally, $\dot{p}^{\prime}: \dot{n} \rightarrow l$ shall be the restriction of $p^{\prime}$ to $\dot{n}$. With these definitions we can state the following

THEOREM. For each $x \in H^{*}(K)$

$$
\left(\mathrm{Sq}^{i} \tau-\tau \mathrm{Sq}^{i}\right) x=d^{*}\left(\sum_{j=0}^{i-2} \dot{p}^{\prime *}\left(\mathrm{Sq}^{j}\left(\varphi^{*} x\right)\right) \alpha^{i-j-1}\right),
$$

where $\tau: H^{*}(K) \rightarrow H^{*}(k)$ is the transfer associated to $\pi: K \rightarrow k$. (Note that we identify $H^{*} L$ with $H^{*} l$.)

The proof uses some results of Bott [1], where he shows that the $\mathrm{Sq}^{i}$ may actually be constructed using a kind of transfer from the Smith sequence of an involution.

3. Suppose that $M \subset K$ is a $Z / 2$-invariant subcomplex and $m$ is its image under $\pi$. Then Smith theory gives us two long exact sequences

$$
\begin{aligned}
& \rightarrow H^{i}(K, M) \stackrel{\Delta^{*}}{\rightarrow} H^{i}(k, m \cup l) \stackrel{\nu}{\rightarrow} H^{i+1}(k, m) \stackrel{\pi^{*}}{\rightarrow} H^{i+1}(K, M) \rightarrow, \\
& \rightarrow H^{i}(K, M \cup L) \stackrel{\Delta^{*}}{\rightarrow} H^{i}(k, m \cup l) \\
& \qquad \stackrel{\mu}{\rightarrow} H^{i+1}(k, m \cup l) \stackrel{\pi^{*}}{\rightarrow} H^{i+1}(K, M \cup L) \rightarrow,
\end{aligned}
$$

which are natural with respect to equivariant simplicial maps between pairs of $\mathbf{Z} / 2$-spaces. In addition, when $M=\varnothing$, the diagram shown in Figure 1 commutes [1].

(The upper horizontal line and right-hand vertical line in the figure are the exact sequences of the pairs $(K, L)$ and $(k, l)$ respectively.) We also have the important fact that $j^{*} \Delta^{*}=\tau$, the ordinary transfer homomorphism for $\pi: K \rightarrow k$ [6].

$$
\begin{aligned}
& H^{i}(k)=H^{i}(k) \\
& \downarrow \pi^{*} \quad \downarrow \\
& H^{i}(K, L) \rightarrow H^{i}(K) \stackrel{\varphi^{*}}{\rightarrow} H^{i}(L) \quad \rightarrow \quad H^{i+1}(K, L) \\
& \left\|\quad \downarrow \Delta^{*} \quad \downarrow \delta^{*} \quad\right\| \\
& H^{i}(K, L) \stackrel{\bar{\Delta}^{*}}{\rightarrow} H^{i}(k, l) \stackrel{\mu}{\rightarrow} H^{i+1}(k, l) \quad \rightarrow \quad H^{i+1}(K, L) \\
& \downarrow \nu \quad \downarrow j^{*} \\
& H^{i+1}(k)=H^{i+1}(k)
\end{aligned}
$$

FIGURE 1 
Bott studies the special case of $K=X \times X$, for $X$ a finite complex. This $K$ is triangulated and subdivided so that the involution $t(x, y)=(y, x)$ becomes a regular action, and the foregoing sequences are applied (with $M=\varnothing$ ). He demonstrates in this case

Proposition [1, p. 588].

$$
\Delta^{*} \mathrm{Sq}^{i}-\mathrm{Sq}^{i} \Delta^{*}=\mu \mathrm{Sq}^{i-1} \Delta^{*}
$$

In fact, this proposition is true for a regular $\mathbf{Z} / 2$-action on any finite complex $K$.

Proof. Consider the map $f: K \rightarrow K \times K$ given by $f(x)=(x, t x)$, where $t \in \mathbf{Z} / 2$ is the generator. If $\mathrm{Z} / 2$ acts on $K \times K$ by the flip involution as above, then $f$ is clearly equivariant. Furthermore, we have $f^{*}(a \times 1)=a$ for each $a \in H^{*}(K)$. Assertion (3) for $K$ now follows from the corresponding statement for $K \times K$, together with the naturality of $f$.

One should note that if $K \times K$ is triangulated so that the diagonal (= fixed point set of the flip involution) is a subcomplex, then $f$ is not generally simplicial. Thus to insure that $f$ is natural, we switch to Čech cohomology [2].

From (3) we get immediately that

$$
\tau \mathrm{Sq}^{i}-\mathrm{Sq}^{i} \tau=j^{*} \mu \mathrm{Sq}^{i-1} \Delta^{*}=\nu \mathrm{Sq}^{i-1} \Delta^{*}
$$

An easy induction, however, then proves that $\mathrm{Sq}^{k} \Delta^{*}=\sum_{j=0}^{k} \mu^{j} \Delta^{*} \mathrm{Sq}^{k-j}$, and so

$$
\tau \mathrm{Sq}^{i}-\mathrm{Sq}^{i} \tau=\nu \sum_{j=0}^{i-1} \mu^{j} \Delta^{*} \mathrm{Sq}^{i-1-j}
$$

The equalities $\mu \Delta^{*}=\delta^{*} \varphi^{*}$ and $j^{*} \delta^{*}=0$ are consequences of the "cross diagram" above and they imply that

$$
\tau \mathrm{Sq}^{i}-\mathrm{Sq}^{i} \tau=j^{*} \sum_{j=0}^{i-1} \mu^{j} \delta^{*} \mathrm{Sq}^{i-j-1} \varphi^{*}=j^{*} \mu \sum_{j=0}^{i-2} \mu^{j} \delta^{*} \mathrm{Sq}^{i-j-2} \varphi^{*}
$$

This, as we shall see, is just the conclusion of our theorem in disguise.

LEMma. Let $M=N, m=n$ in (2), where $N$ and $n$ are the regular neighborhoods described in $\$ 2$. Then we have

$$
j^{*} \mu^{j} \delta^{*} a=d^{*}\left(q^{*} a \alpha^{j}\right),
$$

for each $a \in H^{*}(n \cup l)=H^{*}(n)$. Here $q^{*}: H^{*}(n) \rightarrow H^{*}(\dot{n})$ is the restriction, $\delta^{*}$ : $H^{*}(n) \rightarrow H^{*+1}(k, n)$ and $j^{*}: H^{*}(k, n) \rightarrow H^{*}(k)$.

Proof. To start with, excision yields the following commutative square.

$$
\begin{array}{lcc}
H^{*}(n) & \stackrel{\delta^{*}}{\rightarrow} & H^{*+1}(k, n) \\
q^{*} \downarrow & & i \downarrow \approx \\
H^{*}(\dot{n}) & \stackrel{\delta_{1}}{\rightarrow} & H^{*+1}(k-l, \dot{n})
\end{array}
$$

This, together with naturality in (2), gives $j^{\prime *} \mu^{j} \delta^{*} a=j^{*} i^{-1} \mu^{j} \delta_{1} q^{*} a$. But $\pi$ : $(K-$ $L, \dot{N}) \rightarrow(k-l, \dot{n})$ is a double covering of pairs, since we have removed the fixed 
point set. Therefore (2) reduces in this case to the Smith-Gysin sequence

$$
\rightarrow H^{i}(k-l, \dot{n}) \stackrel{\pi^{*}}{\rightarrow} H^{i}(K-L, \dot{N}) \stackrel{\tau}{\rightarrow} H^{i}(k-l, \dot{n}) \stackrel{\mu}{\rightarrow} H^{i+1}(k-l, \dot{n}) \rightarrow .
$$

Here $\tau$ is the transfer and $\mu(x)=x \beta, \beta \in H^{1}(k-l)$ being the characteristic class of the double cover $(K-L) \rightarrow(k-l)$. Furthermore, $\beta \mid \dot{n}=\alpha$. Therefore,

$$
\begin{aligned}
j^{\prime *} \mu^{j} \delta^{*} a & =j^{\prime *} i^{-1} \mu^{j} \delta_{1} q^{*} a=j^{\prime *} i^{-1} \delta_{1}\left(q^{*} a\right) \beta^{j} \\
& =j^{\prime *} i^{-1} \delta_{1}\left(q^{*} a \alpha^{j}\right)=d^{*}\left(q^{*} a \alpha^{j}\right) .
\end{aligned}
$$

Since $N \cup L=N$ and $n \cup l=n$, we have

$$
\begin{array}{ccccccc}
\rightarrow H^{i}(K, N) & \stackrel{\Delta^{*}}{\rightarrow} & H^{i}(k, n) & \stackrel{\mu}{\rightarrow} & H^{i+1}(k, n) & \stackrel{\pi^{*}}{\rightarrow} & H^{i+1}(K, N) \rightarrow \\
\Gamma^{*} \downarrow \approx & & \gamma^{*} \downarrow \approx & & \gamma^{*} \downarrow \approx & & \Gamma^{*} \downarrow \approx \\
\rightarrow H^{i}(K, L) & \overrightarrow{\Delta^{*}} & H^{i}(k, l) & \stackrel{\mu}{\rightarrow} & H^{i+1}(k, l) & \stackrel{\pi^{*}}{\rightarrow} & H^{i+1}(K, L) \rightarrow
\end{array}
$$

the vertical maps being isomorphisms because $L \subset N$ and $l \subset n$ are homotopy equivalences.

COROLlaRY. $j^{*} \mu^{j} \delta^{*} a=d^{*}\left(\dot{p}^{*} a \alpha^{j}\right)$ for every $a \in H^{*}(l)$, where $\delta^{*}: H^{*}(l) \rightarrow$ $H^{*+1}(k, l)$ and $j^{*}: H^{*}(k, l) \rightarrow H^{*}(k) ; \dot{p}^{\prime}: \dot{n} \rightarrow l$ is the restriction of the retraction $p^{\prime}:$ $n \rightarrow l$.

Proof. Applying the lemma to $p^{* *} a$, we obtain $j^{* *} \mu^{j} \delta^{*} p^{*} a=d^{*}\left(q^{*} p^{*} a \alpha^{j}\right)$. But $j^{*}=j^{*} \gamma^{*}$ and $q^{*} p^{*}=\dot{p}^{*}$, so

$$
d^{*}\left(\dot{p}^{\prime *} a \alpha^{j}\right)=j^{*} \gamma^{*} \mu^{j} \delta^{*} p^{*} a=j^{*} \mu^{j} \gamma^{*} \delta^{*} p^{*} a=j^{*} \mu^{j} \delta^{*} s^{*} p^{*} a
$$

where $s^{*}: H^{*}(n) \rightarrow H^{*}(l)$ is the restriction. Since $p^{\prime}$ is a retraction, it follows that $s^{*} p^{*}=\mathrm{id}$, and thus the corollary is proven.

Applying this corollary to (4), we find that

$$
\begin{aligned}
\left(\tau \mathrm{Sq}^{i}-\mathrm{Sq}^{i} \tau\right) x & =j^{*} \sum_{j=0}^{i-2} \mu^{j+1} \delta^{*} \mathrm{Sq}^{i-j-2} \varphi^{*} x \\
& =d^{*}\left(\sum_{j=0}^{i-2} \dot{p}^{\prime *}\left(\mathrm{Sq}^{i-j-2} \varphi^{*} x\right) \alpha^{j+1}\right) \\
& =d^{*}\left(\sum_{j=0}^{i-2} \dot{p}^{\prime *}\left(\mathrm{Sq}^{j} \varphi^{*} x\right) \alpha^{i-j-1}\right),
\end{aligned}
$$

for each $x \in H^{*}(X)$, so we are done.

4. Since the $\mathrm{Z} / 2$-action involved is only required to be simplicial, we may attempt to use our theorem iteratively for actions of groups more complicated than $\mathbf{Z} / 2$. Namely, suppose $G$ is a 2-group with composition series $G=G_{0} \supset G_{1} \supset \cdots \supset G_{n}$ $=\mathbf{Z} / 2$, and thus $G_{j} / G_{j+1} \approx \mathbf{Z} / 2, j=0, \ldots, n-1$. If $G$ acts simplicially on $X$, then so does each $G_{j}$. Let $\pi_{j}: X \rightarrow X / G_{j}$ be the orbit projection and call the associated 
transfer $\tau_{j}$. Now $G_{j} / G_{j+1} \approx \mathrm{Z} / 2$ acts on $X / G_{j+1}$ and the natural map between orbit spaces $\rho_{j}: X / G_{j+1} \rightarrow X / G_{j}$ is just the orbit projection corresponding to this action. Denote its transfer by $\tau_{j}^{\prime}$. We have $\pi_{j}=\rho_{j} \circ \pi_{j+1}$ and $\tau_{j}=\tau_{j}^{\prime} \circ \tau_{j+1}$. (Note that all of the actions involved are first made regular by taking iterated barycentric subdivisions of $X[2]$.)

In principle, the theorem then yields a formula for $\left(\mathrm{Sq}^{i} \tau_{j}-\tau_{j} \mathrm{Sq}^{i}\right) x$ by downward induction, provided we know $\tau_{k}^{\prime}$ for each $k$ :

$$
\left(\mathrm{Sq}^{i} \tau_{j}-\tau_{j} \mathrm{Sq}^{i}\right) x=\left(\mathrm{Sq}^{i} \tau_{j}^{\prime}-\tau_{j}^{\prime} \mathrm{Sq}^{i}\right)\left(\tau_{j+1} x\right)+\tau_{j}^{\prime}\left(\mathrm{Sq}^{i} \tau_{j+1}-\tau_{j+1} \mathrm{Sq}^{i}\right) x .
$$

On the right-hand side, $\left(\mathrm{Sq}^{i} \tau_{j+1}-\tau_{j+1} \mathrm{Sq}^{i}\right) x$ is known by the inductive hypothesis, and we get $\left(\mathrm{Sq}^{i} \tau_{j}^{\prime}-\tau_{j}^{\prime} \mathrm{Sq}^{i}\right)$ from the theorem, since $\tau_{j}^{\prime}$ arises from an involution. Moreover, we can start the induction since $\tau_{n}$ also comes from a $\mathbf{Z} / 2$-action.

For a general 2-group action, this formula is apt to be quite unwieldy owing to the jumble of different fixed point sets for the various $G_{j} / G_{j+1}$ actions. In special cases, however, we sometimes get simplification.

EXAMPLE. Suppose a 2-group $G$ acts semifreely on $X$, then

$$
\left(\mathrm{Sq}^{i} \tau-\tau \mathrm{Sq}^{i}\right) x=d^{*}\left(\sum_{j=0}^{i-2} \dot{p}^{\prime *} \mathrm{Sq}^{j}\left(\phi^{*} x\right) \tau_{0}^{\prime} \circ \tau_{1}^{\prime} \circ \cdots \circ \tau_{n-1}^{\prime}\left(\alpha^{i-j-1}\right)\right) .
$$

Semifreeness implies that the fixed point set of $G_{n}=F=$ fixed point set of $G$, and that $\pi_{j}(F) \approx F$ is the fixed point set of $G_{j-1} / G_{j}$ for each $j$. Let $n$ be a regular neighborhood of $\pi_{0}(F)$; set $N=\pi_{0}^{-1}(n) \subset X$ and $N^{\prime}=\rho_{n}(N)=$ $\left(\rho_{0} \circ \cdots \circ \rho_{n-1}\right)^{-1}(n) \subset X / G_{n}$. Denote by $\dot{N}, \dot{N}^{\prime}$ and $\dot{n}$ the intersections $(X-F)$ $\cap N,\left(X / G_{n}-F\right) \cap N^{\prime}$ and $(X / G-F) \cap n$. Then $\alpha$ in the above formula is the $\bmod 2$ characteristic class of the double cover $\pi_{n} \mid \dot{N}: \dot{N} \rightarrow \dot{N}^{\prime} ; \dot{p}^{\prime}: \dot{n} \rightarrow F$ is the restriction of the deformation retraction $p^{\prime}: n \rightarrow F \approx \pi_{0}(F)$ and $d^{*}$ is the MayerVietoris coboundary for the triad $(X / G ;(X / G-F), n)$.

The key fact used in demonstrating this example is that

$$
\left(\mathrm{Sq}^{i} \tau_{j}^{\prime}-\tau_{j}^{\prime} \mathrm{Sq}^{i}\right)\left(\tau_{j+1} x\right)=0
$$

in (6) for each $j$. This is so because our theorem asserts that this deviation from commutativity depends on the restriction $\left(\tau_{j+1} x\right) \mid F_{j+1}$, where $F_{j+1}$ is the fixed point set of $G_{j} / G_{j+1}$ in $X / G_{j+1}$. But in this special case $F_{j+1}=F$ and $\left(\tau_{j+1} x\right) \mid F=$ $\tau_{j+1}(x \mid F)=0$, since $\pi_{j+1} \mid F: F \rightarrow F$ is the identity, while $\tau_{j+1} \circ \pi_{j+1}^{*} \equiv 0(\bmod 2)$. This trick actually only requires that $F_{j} \supset F_{j+1}$, so we could use it for an arbitrary action of $\mathbf{Z} / 2^{r}$ on $X$, for example.

The remainder of the example's verification is just a naturality argument. What is important in the resulting formula is that the odd-looking term $\tau_{0}^{\prime} \circ \tau_{1}^{\prime} \circ \cdots \circ \tau_{n-1}^{\prime}\left(\alpha^{i-j-1}\right)$ is really only dependent on the transfer $t: H^{*} G_{n} \rightarrow H^{*} G$ in group cohomology, since $\pi_{0}: \dot{N} \rightarrow \dot{n}$ is a principal $G$-bundle by the semifreeness assumption, and characteristic class data pulls back from $H^{*} B_{G}$. Thus if we know this transfer $t$, the formula in the example is no more complicated than the one in the main theorem. 


\section{BIBLIOGRAPHY}

1. R. Bott, On symmetric products and the Steenrod squares, Ann. of Math. (2) 57 (1953), 579-590.

2. G. E. Bredon, Introduction to compact transformation groups, Academic Press, New York and London, 1972.

3. B. Eckmann, On complexes with operators, Proc. Nat. Acad. Sci. U.S.A. 39 (1953), 35-42.

4. L. Evens, Steenrod operations and transfer, Proc. Amer. Math. Soc. 19 (1968), 1387-1388.

5. S. Illman, Smooth equivariant triangulations of G-manifolds for $G$ a finite group, Math. Ann. 233 (1978), 199-220.

6. R. P. Kubelka, The transfer and Steenrod squares, Dissertation, Stanford University, 1980.

7. R. Schultz, Homological transfers for orbit space projections, Manuscripta Math. 24 (1978), 229-238.

DePartment OF MATHEMATICS, UNIVERSTYY OF OKLAHOMA, NORMAN, OKLAHOMA 73019 